Article

\title{
Symmetry Identities of Changhee Polynomials of Type Two
}

\author{
Joohee Jeong ${ }^{1,+, \ddagger}$, Dong-Jin Kang ${ }^{2, \ddagger}$ and Seog-Hoon Rim ${ }^{1, *, \ddagger}$ \\ 1 Department of Mathematics Education, Kyungpook National University, Daegu 41566, Korea; \\ jhjeong@knu.ac.kr \\ 2 Department of Computer Engineering, Information Technology Services, Kyungpook National University, \\ Daegu 41566, Korea; djkang@knu.ac.kr \\ * Correspondence: shrim@knu.ac.kr; Tel.: +82-53-950-5890 \\ + Current address: 80 Daehakro, Bukgu, Daegu 41566, Korea. \\ $\ddagger$ These authors contributed equally to this work.
}

Received: 31 October 2018 ; Accepted: 6 December 2018 ; Published: 11 December 2018

check for updates

\begin{abstract}
In this paper, we consider Changhee polynomials of type two, which are motivated from the recent work of D. Kim and T. Kim. We investigate some symmetry identities for the Changhee polynomials of type two which are derived from the properties of symmetry for the fermionic $p$-adic integral on $\mathbb{Z}_{p}$.
\end{abstract}

Keywords: Changhee polynomials; Changhee polynomials of type two; fermionic $p$-adic integral on $\mathbb{Z}_{p}$

\section{Introduction}

Let $p$ be a fixed odd prime number. Throughout this paper, $\mathbb{Z}_{p}, \mathbb{Q}_{p}$ and $\mathbb{C}_{p}$ will denote the ring of $p$-adic integers, the field of $p$-adic rational numbers and the completion of the algebraic closure of $\mathbb{Q}_{p}$.

The $p$-adic norm $|\cdot|_{p}$ is normalized as $|p|_{p}=\frac{1}{p}$.

Let $f(x)$ be a continulus funciton on $\mathbb{Z}_{p}$. Then the fermionic $p$-adic integral on $\mathbb{Z}_{p}$ is defined by Kim in [1] as

$$
\int_{\mathbb{Z}_{p}} f(x) d \mu_{-1}(x)=\lim _{N \rightarrow \infty} \sum_{x=0}^{p^{N}-1} f(x) \mu_{-1}(x)=\lim _{x \rightarrow \infty} \sum_{x=0}^{p^{N}-1} f(x)(-1)^{x} .
$$

For $n \in \mathbb{N}$, by (1), we get

$$
\begin{array}{r}
\int_{\mathbb{Z}_{p}} f(x+n) d \mu_{-1}(x)+(-1)^{n-1} \int_{\mathbb{Z}_{p}} f(x) d \mu_{-1}(x) \\
=2 \sum_{\ell=0}^{n-1} f(\ell)(-1)^{n-1-\ell}
\end{array}
$$

as shown in [2-5]. In particular, if we take $n=1$, then we have

$$
\int_{\mathbb{Z}_{p}} f(x+1) d \mu_{-1}(x)+\int_{\mathbb{Z}_{p}} f(x) d \mu_{-1}(x)=2 f(0),
$$

which is noted in [6,7]. 
In the previous paper [8], D. Kim and T. Kim introduced the Changhee polynomials $\widetilde{C h}_{n}(x)$ of type two by the generating function

$$
\sum_{n=0}^{\infty} \widetilde{C h}_{n}(x) \frac{t^{n}}{n !}=\frac{2}{(1+t)+(1+t)^{-1}}(1+t)^{x} .
$$

By exploiting the method of fermionic $p$-adic integral on $\mathbb{Z}_{p}$, the Changhee polynomials of type two can be represented by the fermionic $p$-adic integrals of $\mathbb{Z}_{p}$ : for $t \in \mathbb{C}_{p}$ with $|t|_{p}<p^{-\frac{1}{p-1}}$,

$$
\begin{aligned}
\int_{\mathbb{Z}_{p}}(1+t)^{2 y+1+2 x} d \mu_{-1}(y) & =\frac{2}{(1+t)^{2}+1}(1+t)^{2 x+1} \\
& =\sum_{n=0}^{\infty} \widetilde{C} h_{n}(x) \frac{t^{n}}{n !}
\end{aligned}
$$

When $x=0, \widetilde{C h}_{n}=\widetilde{C h}_{n}(0)$ are called the Changhee numbers of type two.

In this paper, we will introduce further generalization of Changhee polynomials of type two, by using again fermionic $p$-adic integration on $\mathbb{Z}_{p}$.

We investigate some symmetry identities for the $w$-Changhee polynomials of type two which are derived from the properties of symmetry for the fermionic $p$-adic integral on $\mathbb{Z}_{p}$. Many authors investigated symmetric properties of special polynomials and numbers. See [9-12] and their references.

We introduce $w$-Changhee polynomials of type two in Section 3.

\section{Changhee Polynomials and Numbers of Type Two}

In this section, we use the techniques presented in the articles of C. Cesarano, C. Fornaro [13] and C. Cesarno [14], in particular the similarity of Chebyshev polynomials.

By using the generating functions of Changhee numbers and polynomials of type two, we have the following result.

Proposition 1. For $n \in \mathbb{N}$ and $1 \leq k \leq n$, we have

$$
\widetilde{C h}_{n}(x)=\sum_{m=0}^{n}\left(\begin{array}{l}
n \\
m
\end{array}\right)(2 x)_{m} \widetilde{C h}_{n-m}
$$

where $(x)_{n}=x(x-1) \cdots(x-n+1),(n \geq 1),(x)_{0}=1$.

\section{Proof of Proposition 1.}

$$
\begin{aligned}
\sum_{n=0}^{\infty} \widetilde{C h}_{n}(x) \frac{t^{n}}{n !} & =\frac{2}{(1+t)+(1+t)^{-1}}(1+t)^{2 x} \\
& =\sum_{m=0}^{\infty} \widetilde{C h_{m}} \frac{t^{m}}{m !} \sum_{\ell=0}^{\infty}(2 x)_{\ell} \frac{t^{n}}{\ell !} \\
& =\sum_{n=0}^{\infty}\left(\sum_{m=0}^{n}\left(\begin{array}{c}
n \\
m
\end{array}\right) \widetilde{C h}_{m}(2 x)_{n-m}\right) \frac{t^{n}}{n !}
\end{aligned}
$$

The Stirling number $S_{1}(\ell, n)$ of the first kind is defined in $[2-5,15]$ by the generating function

$$
(\log (1+t))^{n}=n ! \sum_{\ell=n}^{\infty} S_{1}(\ell, n)
$$


and the Stirling number $S_{2}(m, n)$ of the second kind is given in [4] by the generating function

$$
\left(e^{t}-1\right)^{n}=n ! \sum_{m=n}^{\infty} S_{2}(m, n) \frac{t^{m}}{m !} .
$$

As is well known, the Euler polynomials $E_{n}(x)$ are defined in [16-18] by the generating function

$$
\frac{2}{e^{t}+1} e^{x t}=\sum_{n=0}^{\infty} E_{n}(x) \frac{t^{n}}{n !} .
$$

When $x=0, E_{n}=E_{n}(0),(n \geq 0)$, are called the $n$-th Euler numbers, whereas the Euler numbers $E_{n}^{*}$ of the second kind are given by the generating function

$$
\operatorname{sech}(t)=\frac{2}{e^{t}+e^{-t}}=\sum_{n=0}^{\infty} E_{n}^{*} \frac{t^{n}}{n !}
$$

as noted in [16,19].

Before we proceed, we study some relevant relations between the Changhee numbers of type two and the Euler numbers of the second kind.

Proposition 2. For $n \in \mathbb{N}$ and $0 \leq k \leq n$, we have

$$
\widetilde{C h}_{n}=\sum_{k=0}^{n} E_{k}^{*} S_{1}(n, k) .
$$

Proof of Proposition 2. From the generating functions of Changhee numbers of type two shown in (8), we have

$$
\begin{aligned}
\sum_{n=0}^{\infty} \widetilde{C h_{n}} \frac{t^{n}}{n !} & =\frac{2}{(1+t)+(1+t)^{-1}}=\frac{2}{e^{\log (1+t)}+e^{-\log (1+t)}} \\
& =\operatorname{sech}(\log (1+t)) \\
& =\sum_{n=0}^{\infty} E_{n}^{*} \frac{(\log (1+t))^{n}}{n !}=\sum_{n=0}^{\infty}\left(\sum_{k=0}^{n} E_{k}^{*} S_{1}(n, k)\right) \frac{t^{n}}{n !} .
\end{aligned}
$$

Thus we have the result.

The result above helps us to derive some values of Changhee numbers of type two $\widetilde{C h}_{n}$ 's as follows: from $E_{0}^{*}=1, E_{1}^{*}=0, E_{2}^{*}=-1, E_{3}^{*}=0, E_{4}^{*}=5, E_{5}^{*}=0$ and $S_{1}(n, n)=0$ for $n \geq 0, S_{1}(n, 0)=0$ for $n \geq 1, S_{1}(2,1)=1, S_{1}(3,1)=2, S_{1}(4,1)=6, S_{1}(5,1)=24, S_{1}(3,2)=3, S_{1}(4,2)=11, S_{1}(5,2)=50$, $S_{1}(4,3)=6, S_{1}(5,3)=35, S_{1}(5,4)=10$,

$$
\begin{aligned}
& \widetilde{C h_{0}}=E_{0}^{*} S_{1}(0,0)=1, \\
& \widetilde{C h_{1}}=E_{0}^{*} S_{1}(1,0)+E_{1}^{*} S_{1}(1,1)=0+0=0, \\
& \widetilde{C h_{2}}=E_{0}^{*} S_{1}(2,0)+E_{1}^{*} S_{1}(2,1)+E_{2}^{*} S_{1}(2,2)=0+0-1=-1 . \\
& \widetilde{C h}_{3}=E_{0}^{*} S_{1}(3,0)+E_{1}^{*} S_{1}(3,1)+E_{2}^{*} S_{1}(3,2)+E_{3}^{*} S_{1}(3,3) \\
& \quad=0+0-3+0=-3, \\
& \widetilde{C h_{4}}=E_{0}^{*} S_{1}(4,0)+E_{1}^{*} S_{1}(4,1)+E_{2}^{*} S_{1}(4,2)+E_{3}^{*} S_{1}(4,3)+E_{4}^{*} S_{1}(4,4) \\
& \quad=0+0-11+0+5=-6, \\
& \widetilde{C h}_{5}=E_{0}^{*} S_{1}(5,0)+E_{1}^{*} S_{1}(5,1)+E_{2}^{*} S_{1}(5,2)+E_{3}^{*} S_{1}(5,3)+E_{4}^{*} S_{1}(5,4) \\
& \quad+E_{5}^{*} S_{1}(5,5)=0+0-50+0+50+0=0 .
\end{aligned}
$$


For the inversion formulas for Proposition 2, we have the following.

Proposition 3. For $n \in \mathbb{N}$ and $0 \leq k \leq n$, we have

$$
E_{n}^{*}=\sum_{k=0}^{n} \widetilde{C h}_{k} S_{2}(n, k)
$$

Proof of Proposition 3. From (6) and (8), we get the following, by replacing $t$ by $e^{t}-1$ :

$$
\begin{aligned}
\frac{2}{(1+t)^{2}+1}(1+t) & =\sum_{n=0}^{\infty} \widetilde{C h_{n}} \frac{t^{n}}{n !} \\
\frac{2}{e^{2 t}+1} e^{t} & =\sum_{k=0}^{\infty} \widetilde{C h_{k}} \frac{1}{k !}\left(e^{t}-1\right)^{k} \\
& =\sum_{n=0}^{\infty}\left(\sum_{k=0}^{n} \widetilde{C h}_{k} S_{2}(n, k)\right) \frac{t^{n}}{n !} \\
& =\frac{2}{e^{t}+e^{-t}}=\sum_{n=0}^{\infty} E_{n}^{*} \frac{t^{n}}{n !} .
\end{aligned}
$$

Now (11) gives us the desired result $E_{n}^{*}=\sum_{k=0}^{n} \widetilde{C} h_{k} S_{2}(n, k)$.

Also by using the fermionic $p$-adic integration on $\mathbb{Z}_{p}$, we can represent Changhee numbers of type two as follows.

Proposition 4 (Witt's formula for Changhee numbers of type two).

For $n \in \mathbb{N}$, we have

$$
\widetilde{C h_{n}}=\int_{\mathbb{Z}_{p}}(2 x+1)_{n} d \mu_{-1}(x)
$$

Proof of Proposition 4. First, we observe

$$
\begin{aligned}
\int_{\mathbb{Z}_{p}}(1+t)^{2 x+1} d \mu_{-1}(x) & =\int_{\mathbb{Z}_{p}} \sum_{n=0}^{\infty}(2 x+1)_{n} \frac{t^{n}}{n !} d \mu_{-1}(x) \\
& =\sum_{n=0}^{\infty} \int_{\mathbb{Z}_{p}}(2 x+1)_{n} d \mu_{-1} \frac{t^{n}}{n !},
\end{aligned}
$$

On the other hand, by the definition of fermionic $p$-adic integration on $\mathbb{Z}_{p}$,

$$
\int_{\mathbb{Z}_{p}}(1+t)^{2 x+1} d \mu_{-1}(x)=\frac{2}{(1+t)^{2}+1}(1+t)=\sum_{n=0}^{\infty} \widetilde{C h_{n}} \frac{t^{n}}{n !} .
$$

Thus, by comparing the coefficients of both sides of (13) and (14), we have the desired result.

\section{Symmetry of $w$-Changhee Polynomials of Type Two}

Motivated from D. Kim and T. Kim [20], for $w \in \mathbb{N}$, we define $w$-Changhee polynomials of type two by the following generating function

$$
\frac{2}{(1+t)^{2 w}+1}(1+t)^{2 w x+1}=\sum_{n=0}^{\infty} \widetilde{C h}_{n, w}(x) \frac{t^{n}}{n !} .
$$

When $x=0, \widetilde{C h}_{n, w}=\widetilde{C h}_{n, w}(0)$ are called the $w$-Changhee numbers of type two. When $w=1$, $\widetilde{C h}_{n, 1}(x)=\widetilde{C h}_{n}(x)$ are just the Changhee polynomials of type two in (4). For the case of $w=\frac{1}{2}$, 
the $\frac{1}{2}$-Changhee polynomials of type two are related to the well-known Changhee polynomials of type two, i.e., $\widetilde{C h}_{n, \frac{1}{2}}(x)=\widetilde{C h}_{n}(x+1)$.

The generating function of $w$-Changhee polynomials of type two can be related with Changhee polynomials of type two or Changhee numbers of type two as follows.

Proposition 5. For $n, w, \ell \in \mathbb{N}$ and $1 \leq \ell \leq n$, we have

$$
\begin{aligned}
& \text { (1) } \widetilde{C h}_{n, w}(x)=\sum_{\ell=0}^{n} \widetilde{C h}_{\ell}(2 w x) \text {, and } \\
& \text { (2) } \widetilde{C h}_{n, w}(x)=\sum_{\ell=0}^{n}\left(\begin{array}{l}
n \\
\ell
\end{array}\right)(2 w x)_{\ell} \widetilde{C h}_{n-\ell} .
\end{aligned}
$$

Proof of Proposition 5. (1) is immediate from the definition. For (2), we have

$$
\begin{aligned}
\sum_{n=0}^{\infty} \widetilde{C h}_{n, w}(x) \frac{t^{n}}{n !} & =\left(\sum_{\ell=0}^{\infty} \widetilde{C h}_{\ell} \frac{t^{\ell}}{\ell !}\right)(1+t)^{2 w x} \\
& =\left(\sum_{\ell=0}^{\infty} \widetilde{C h}_{\ell} \frac{t^{\ell}}{\ell !}\right)\left(\sum_{m=0}^{\infty}(2 w x)_{m} \frac{t^{m}}{m !}\right) \\
& \left.=\sum_{n=0}^{\infty}\left(\sum_{\ell=0}^{n}\left(\begin{array}{l}
n \\
\ell
\end{array}\right)(2 w x)_{\ell} \widetilde{C h}_{n-\ell}\right\}\right) \frac{t^{n}}{n !} .
\end{aligned}
$$

From (3), we can easily derive the following:

$$
2 \sum_{\ell=0}^{n}(-1)^{\ell}(1+t)^{2 \ell}=\frac{2\left\{1+(-1)^{n+1}(1+t)^{2(n+1)}\right\}}{(1+t)^{2}+1}
$$

The left hand side of (16) can be written as

$$
2 \sum_{\ell=0}^{n}(-1)^{\ell}(1+t)^{2 \ell}=\sum_{n=0}^{\infty}\left(\sum_{\ell=0}^{n-1}(-1)^{\ell}(2 \ell)_{n}\right) \frac{t^{n}}{n !}
$$

We use the notation of $\lambda$-falling factorial in $[12,21]$ for $\lambda \in \mathbb{R}$,

$$
(\ell \mid \lambda)_{n}= \begin{cases}\ell(\ell-\lambda) \cdots(\ell-\lambda(n-1)), & (\text { if } n \geq 1) \\ 1, & (\text { if } n=0) .\end{cases}
$$

Then the right hand side of (17) can be written as

$$
2 \sum_{\ell=0}^{n-1}(-1)^{\ell}(1+t)^{2 \ell}=\sum_{n=0}^{\infty} T_{m}\left(n ;\left(\ell \mid \frac{1}{2}\right)\right) \frac{t^{n}}{n !} .
$$

where we denote, for $\lambda \in \mathbb{R}$,

$$
T_{m}(n ;(\ell \mid \lambda))=\sum_{\ell=0}^{n}(-1)^{\ell}(\ell \mid \lambda)_{m} .
$$

For $n \in \mathbb{N}, n \equiv 1(\bmod 2), m \geq 0$ we have

$$
\sum_{m=0}^{\infty} 2\left(\sum_{\ell=0}^{n}(-1)^{\ell}(-2 \ell)_{m}\right) \frac{t^{m}}{m !}=\frac{2\left(1+(1+t)^{2(n+1)}\right)}{(1+t)^{2}+1} .
$$


On the other hand, by (4) and (18), we have

$$
\begin{aligned}
\sum_{m=0}^{\infty}\left(\widetilde{C h}_{m}+\widetilde{C h}_{m}(n+1)\right) \frac{t^{m}}{m !} & =\frac{2(1+t)}{(1+t)^{2}+1}+\frac{2(1+t)^{2(n+1)}(1+t)}{(1+t)^{2}+1} \\
& =2 \sum_{\ell=0}^{n}(-1)^{\ell}(1+t)^{2 \ell+1} \\
& =2 T_{m}\left(n ;\left(\ell+\frac{1}{2} \mid \frac{1}{2}\right)\right) .
\end{aligned}
$$

Now we consider a quotient of fermionic $p$-adic integrals on $\mathbb{Z}_{p}$,

$$
\begin{aligned}
\frac{2 \int_{\mathbb{Z}_{p}}(1+t)^{2 w_{2} x_{2}} d \mu_{-1}\left(x_{2}\right)}{\int_{\mathbb{Z}_{p}}(1+t)^{2 w_{1} w_{2} x_{1}} d \mu_{-1}\left(x_{1}\right)} & =\sum_{\ell=0}^{w_{1}-1}(-1)(1+t)^{2 w_{2} \ell} \\
& =\sum_{m=0}^{\infty} \sum_{\ell=0}^{w_{1}-1}(-1)^{\ell}\left(2 w_{2} \ell\right)_{m} \\
& =\sum_{m=0}^{\infty} \sum_{\ell=0}^{w_{1}-1}\left(2 w_{2}\right)^{m}(-1)^{\ell}\left(\ell \mid \frac{1}{2 w_{2}}\right)_{m} \\
& =\sum_{m=0}^{\infty}\left(2 w_{2}\right)^{m} T_{m}\left(w_{1}-1 \mid\left(\ell \mid \frac{1}{2 w_{2}}\right)\right),
\end{aligned}
$$

where $T_{m}(n \mid(\ell \mid \lambda))=\sum_{\ell=0}^{n}(-1)^{\ell}(\ell \mid \lambda)_{m}$ for $\lambda \in \mathbb{R}$.

For the symmetry of $w$-Changhee polynomials of type two, we consider the following quotient form of fermionic $p$-adic integration on $\mathbb{Z}_{p}$.

$$
\begin{aligned}
T\left(w_{1}, w_{2}\right)= & \frac{2 \int_{\mathbb{Z}_{p}} \int_{\mathbb{Z}_{p}}(1+t)^{2 w_{1} x_{1}+2 w_{2} x_{2}+2} d \mu_{-1}\left(x_{1}\right) d \mu_{-1}\left(x_{2}\right)}{\int_{\mathbb{Z}_{p}}(1+t)^{2 w_{1} w_{2} x_{1}+1} d \mu_{-1}\left(x_{1}\right)}(1+t)^{2 w_{1} w_{2} x} \\
= & \int_{\mathbb{Z}_{p}}(1+t)^{2 w_{1} x_{1}+1} d \mu_{-1}\left(x_{1}\right)(1+t)^{2 w_{1} w_{2} x} \\
& \times \frac{\int_{\mathbb{Z}_{p}}(1+t)^{2 w_{2} x_{2}} d \mu_{-1}\left(x_{2}\right)}{\int_{\mathbb{Z}_{p}}(1+t)^{2 w_{1} w_{2} x_{1}} d \mu_{-1}\left(x_{1}\right)} \\
= & \left(\sum_{\ell=0}^{\infty} \widetilde{C h}_{\ell, w_{1}}\left(w_{2} x\right) \frac{t^{\ell}}{\ell !}\right)\left(\sum_{k=0}^{\infty}\left(2 w_{2}\right)^{k} T_{k}\left(w_{1}-1 \mid\left(k \mid \frac{1}{2 w_{2}}\right)\right)\right) \\
= & \sum_{n=0}^{\infty}\left(\sum_{k=0}^{n}\left(\begin{array}{l}
n \\
k
\end{array}\right) \widetilde{C h_{n-k, w_{1}}}\left(w_{2} x\right)\left(2 w_{k}\right)^{k} T_{k}\left(w_{1}-1 \mid\left(k \mid \frac{1}{2 w_{2}}\right)\right)\right) \frac{t^{n}}{n !} .
\end{aligned}
$$
and $w_{2}$.

Similarly we have the following identity for $T\left(w_{1}, w_{2}\right)$ because $T\left(w_{1}, w_{2}\right)$ is symmetric on $w_{1}$

$$
T\left(w_{1}, w_{2}\right)=\sum_{n=0}^{\infty}\left(\sum_{k=0}^{n}\left(\begin{array}{l}
n \\
k
\end{array}\right) \widetilde{C h}_{n-k, w_{2}}\left(w_{1} x\right)\left(2 w_{1}\right)^{k} T_{k}\left(w_{2}-1 \mid\left(k \mid \frac{1}{2 w_{1}}\right)\right)\right) \frac{t^{n}}{n !} .
$$

Thus, by (22) and (23), we have the following theorem.

Theorem 1. For $w_{1}, w_{2} \in \mathbb{N}$ with $w_{1} \equiv 1(\bmod 2), w_{2} \equiv 1(\bmod 2)$ and $n \geq 0$, we have

$$
\begin{aligned}
& \sum_{k=0}^{n}\left(\begin{array}{l}
n \\
k
\end{array}\right) \widetilde{C h}_{n-k, w_{2}}\left(w_{1} x\right)\left(2 w_{1}\right)^{k} T_{k}\left(w_{2}-1 \mid\left(k \mid \frac{1}{2 w_{1}}\right)\right) \\
& =\sum_{k=0}^{n}\left(\begin{array}{l}
n \\
k
\end{array}\right) \widetilde{C h}_{n-k, w_{1}}\left(w_{2} x\right)\left(2 w_{2}\right)^{k} T_{k}\left(w_{1}-1 \mid\left(k \mid \frac{1}{2 w_{2}}\right)\right) .
\end{aligned}
$$


If we take $w_{2}=1$ in Theorem 1 , we have the following

Corollary 1. For $w_{1} \in \mathbb{N}$ with $w_{1} \equiv 1(\bmod 2)$ and $n \geq 0$, we have

$$
\widetilde{C h}_{n}\left(w_{1} x\right)=\sum_{k=0}^{n}\left(\begin{array}{l}
n \\
k
\end{array}\right) \widetilde{C h}_{n-k, w_{1}}(x) 2^{k} T_{k}\left(w_{1}-1 \mid\left(k \mid \frac{1}{2}\right)\right) .
$$

From (22), we rewrite $T\left(w_{1}, w_{2}\right)$ as follows:

$$
\begin{aligned}
T\left(w_{1}, w_{2}\right)= & \int_{\mathbb{Z}_{p}}(1+t)^{2 w_{1} x_{1}} d \mu_{-1}\left(x_{1}\right)(1+t)^{2 w_{1} w_{2} x} \\
& \quad \times \frac{2 \int_{\mathbb{Z}_{p}}(1+t)^{2 w_{2} x_{2}} d \mu_{-1}\left(x_{2}\right)}{\int_{\mathbb{Z}_{p}}(1+t)^{2 w_{1} w_{2} x_{1}} d \mu_{-1}\left(x_{1}\right)} \\
= & \int_{\mathbb{Z}_{p}}(1+t)^{2 w_{1} x_{1}} d \mu_{-1}\left(x_{1}\right)(1+t)^{2 w_{1} w_{2} x} \\
& \quad \times 2 \sum_{\ell=0}^{w_{1}-1}(1+t)^{2 w_{2} \ell}(-1)^{\ell} \\
= & 2 \sum_{\ell=0}^{w_{1}-1}(-1)^{\ell} \int_{\mathbb{Z}_{p}}(1+t)^{2 w_{1} x_{1}+2 w_{1} w_{2} x+2 w_{2} \ell} d \mu_{-1}\left(x_{1}\right) \\
= & 2 \sum_{\ell=0}^{w_{1}-1}(-1)^{\ell} \int_{\mathbb{Z}_{p}}(1+t)^{2 w_{1} x_{1}+2 w_{1} w_{2} x+\frac{w_{2}}{w_{1}} \ell} d \mu_{-1}\left(x_{1}\right) \\
= & 2 \sum_{\ell=0}^{w_{1}-1}(-1)^{\ell} \sum_{k=0}^{\infty} \widetilde{C h_{k, w_{1}}}\left(w_{2} x+\frac{w_{2}}{w_{1}} \ell\right) \frac{t^{k}}{k !} \\
= & \sum_{n=0}^{\infty}\left(2 \sum_{\ell=0}^{w_{1}-1}(-1)^{\ell} \widetilde{C h}_{n, w_{1}}\left(\frac{w_{2}}{w_{1}} \ell+w_{2} x\right)\right) \frac{t^{n}}{n !}
\end{aligned}
$$

Similarly, by the symmetry of $T\left(w_{1}, w_{2}\right)$, we have the following identity

$$
T\left(w_{1}, w_{2}\right)=\sum_{n=0}^{\infty}\left(2 \sum_{\ell=0}^{w_{2}-1}(-1)^{\ell} \widetilde{C}_{n, w_{2}}\left(\frac{w_{1}}{w_{2}} \ell+w_{1} x\right)\right) \frac{t^{n}}{n !} .
$$

Now from (24) and (25), we have the following theorem.

Theorem 2. For $w_{1}, w_{2} \in \mathbb{N}$ with $w_{1} \equiv 1(\bmod 2), w_{2} \equiv 1(\bmod 2)$ and $n \geq 0$, we have

$$
\sum_{\ell=0}^{w_{1}-1}(-1)^{\ell} \widetilde{C h}_{n, w_{1}}\left(\frac{w_{2}}{w_{1}} \ell+w_{2} x\right)=\sum_{\ell=0}^{w_{2}-1}(-1)^{\ell} \widetilde{C h}_{n, w_{2}}\left(\frac{w_{1}}{w_{2}} \ell+w_{1} x\right) .
$$

When we take $w_{2}=1$, we have

$$
\widetilde{C h}_{n}\left(w_{1} \ell+w_{1} x\right)=\sum_{\ell=0}^{w_{1}-1}(-1)^{\ell} \widetilde{C h}_{n, w_{1}}\left(\frac{\ell}{w_{1}}+x\right) .
$$

\section{Conclusions}

The Changhee polynomials of type two are considered by D. Kim and T. Kim (see [8]) and various properties on their polynomials and numbers are investigated.

In this paper, we investigate some symmetry identities for the Changhee polynomials of type two which are derived from the properties of symmetry for the fermionic $p$-adic integrals on $\mathbb{Z}_{p}$. The techniques presented in the articles by Cesarano and Fornaro $[13,14]$, paticularly the Chebyshev polynomials, are used.

Especially we introduce $w$-Changhee polynomials of type two and investigate interesting symmetry identities. 
For the cases of $w=1, w=\frac{1}{2}$ and $w=\frac{1}{4}$, the symmetry of the $w$-Changhee polynomials of type two are related to the works of Changhee polynomials of type two, those of well-known Changhee polynomials (see [4,22]), and those of the Catalan polynomials (see [20]) respectively.

Recently, many works are done on some identities of special polynomials in the view point of degenerate sense (see $[15,20,21])$. Our result could be developed in that direction also: i.e., on the symmetry of the degenerate $w$-Changhee polynomials of type two.

Finally, we remark that our results on symmetry of two variables could be extended to the three variables case.

Author Contributions: All authors contributed equally to this work. All authors read and approved the final manuscript.

Funding: This research received no external funding.

Acknowledgments: The authors would like to thank the referees for their valuable comments which improved the original manuscript in its present form.

Conflicts of Interest: The authors declare no conflict of interest.

\section{References}

1. Kim, T. q-Volkenborn integration. Russ. J. Math. Phys. 2002, 9, 288-299.

2. Kim, D.S. Identities associated with generalized twisted Euler polynomials twisted by ramified roots of unity. Adv. Stud. Contemp. Math. (Kyungshang) 2012, 22, 363-377.

3. Kim, D.S.; Kim, T.; Kim, Y.H.; Lee, S.H. Some arithmetic properties of Bernoulli and Euler numbers. Adv. Stud. Contemp. Math. (Kyungshang) 2012, 22, 467-480.

4. Kim, D.S.; Kim, T.; Seo, J.J. A note on Changhee polynomials and numbers. Adv. Stud. Theor. Phys. 2013, 7, 993-1003. [CrossRef]

5. Kim, T.; Rim, S.-H. New Changhee $q$-Euler numbers and polynomials associated with $p$-adic $q$-integerals. Comput. Math. Appl. 2007, 54, 484-489. [CrossRef]

6. Kim, T. Non Archmedean $q$-integrals associated with multiple Changhee $q$-Bernoulli polynomials. Russ. Math. Phys. 2003, 10, 91-98.

7. Kim, T. $p$-adic $q$-integrals associated with the Changhee-Barnes' $q$-Bernoulli polynomials. Integr. Transf. Spec. Funct. 2004, 15, 415-420. [CrossRef]

8. Kim, D.S.; Kim, T. A note on type 2 Changhee and Daehee polynomials. arXiv 2018, arXiv:1809.05217.

9. Kim, D.S.; Lee, N.; Na, H.; Park, K.H. Abundant symmetry for higher-order Bernoulli polynomials (I). Adv. Stud. Contemp. Math. 2013, 23, 461-482.

10. Kim, D.S.; Lee, N.; Na, J.; Park, K.H. Abundant symmetry for higher-order Bernoulli polynomials (II). Proc. Jangjeon Math. Soc. 2013, 16, 359-378.

11. Kim, T. Symmetry $p$-adic invariant integral on $\mathbb{Z}_{p}$ for Bernoulli and Euler polynomials. J. Differ. Equ. Appl. 2008, 14, 1267-1277. [CrossRef]

12. Kim, T.; Dolgy, D. On the identities of symmetry for degenerate Bernoulli polynomials of order $r$. Adv. Stud. Contemp. Math. 2015, 25, 457-462.

13. Cesarano, C.; Fornaro, C. A note on two-variable Chebyshev polynomials. Georgian Math. J. 2017, 24, 339-349. [CrossRef]

14. Cesarno, C. Generalized Chebyshev polynomials. Hacet. J. Math. Stat. 2014, 43, 731-740.

15. Kim, T.; Kim, D.S. Identities for degenerate Bernoulli polynomials and Korobov polynomials of the first kind. Sci. China Math. 2018. [CrossRef]

16. Kim, D.S.; Kim, T. Some $p$-adic integrals on $\mathbb{Z}_{p}$ associated with trigonometric functions. Russ. J. Math. Phys. 2018, 25, 300-308. [CrossRef]

17. Simsek, Y. Identities on Changhee numbers and Apostol-type Daehee polynomials. Adv. Stud. Contemp. Math. (Kyungshang) 2017, 27, 199-212.

18. Zhang, W.P. Number of solutions to a congruence equations mod p. J. Northwest Univ. Natl. Sci. 2016, 46, 313-316. (In Chinese)

19. Knuth, D.E.; Buckholtz, T.J. Computation of Tangent, Euler, and Bernoulli Numbers. Math. Comput. 1967, 21, 663-688. [CrossRef] 
20. Kim, D.S.; Kim, T. Triple symmetric identities for $w$-Caralan polynomials. J. Korean Math. Soc. 2017, 54, 1243-1264.

21. Kim, T.; Kim, D.S. Identities of symmetry for degenerate Euler polynomials and alternating generalized falling factorial sums. Iran. J. Sci. Technol. Trans. A Sci. 2017, 41, 939-949. [CrossRef]

22. Kim, T. Symmetry of power sum polynomials and multivariate fermionic $p$-adic invariant integral on $\mathbb{Z}_{p}$. Russ. J. Math. Phys. 2009, 16, 93-96. [CrossRef]

(C) 2018 by the authors. Licensee MDPI, Basel, Switzerland. This article is an open access article distributed under the terms and conditions of the Creative Commons Attribution (CC BY) license (http://creativecommons.org/licenses/by/4.0/). 